\title{
The beast behind the dwarf
}

\section{Cosmina Rimbu1 ${ }^{1}$, Lisandra Damian ${ }^{1,2}$, Letitia Leustean ${ }^{1,2}$, Ingrith Miron ${ }^{2,3}$, Alexandru}

Florescu $^{2}$, Carmen Vulpoi, ${ }^{1,2}$, Maria Christina Ungureanu ${ }^{1,2}$.

1. Endocrinology Clinic, "Sf. Spiridon " Emergency Clinical Hospital, Iasi,Romania.

2." Grigore T. Popa", University of Medicine and Pharmacy , Iasi,Romania.

3. $4^{\text {th }}$ Pediatric Clinic, "Sf. Maria " Clinical Hospital, Iasi, Romania.

\section{Introduction:}

Case report
Abnormalities of growth are one of the most common reasons for the pediatric-endocrinology consults. It's an obvious manifestation with countless possible causes behind, and sometimes we can have unexpected diagnosis.
Clinical evaluation:

- $89,5 \mathrm{~cm}(-4 \mathrm{SD})$,

- $11 \mathrm{~kg}(-2,5 \mathrm{SD})$,

- discreet ocular asymmetry.
We investigated the case of a 4 years old girl, born at term, naturally, SGA : birth weight $=1950 \mathrm{~g}$, who presented in the Endocrinology Department for short stature.
Moreover, the first day of admission we noted polyuria and polydipsia not reported by mother: $>$ ingestion $3750 \mathrm{ml} / 24 \mathrm{~h}$,

$>$ excretion $3950 \mathrm{ml} / 24 \mathrm{~h}$.

The suspicion of diabetes insipidus was confirmed.
- normal karyotype

- normal thoracic X-ray and abdominal ultrasound.
- $\mathrm{ACTH}=36 \mathrm{pg} / \mathrm{ml}(0-46)$

- cortisol= $14 \mathrm{ug} / \mathrm{dl}(5-25)$

- normal thyroid function:

- TSH=0,849 uIU/ml (0,3- 6,3 )

- FT4=1,25 ng/dl $(0,89-1,76)$. and benign, the histiocytosis was found to be an unexpected and unpleasant diagnosis which involves more aggressive treatment and complications.

The patient received treatment for anemic syndrome and for diabetes insipidus ( Desmopressin $30 \mathrm{ug} / \mathrm{day}$ ). She was directed to the Pediatric Clinic where chemotherapy was initiated. 